\title{
Altura da garupa e sua associação com características reprodutivas e de crescimento na raça Nelore
}

\author{
Marcio Cinachi Pereira ${ }^{(1)}$, Marcos Juniti Yokoo ${ }^{(2)}$, Annaiza Braga Bignardi ${ }^{(2)}$, \\ Juliana Cristina Sezana ${ }^{(3)}$ e Lucia Galvão de Albuquerque ${ }^{(2)}$
}

\begin{abstract}
(1)Embrapa Gado de Leite, Rua Eugênio do Nascimento, no 610, Dom Bosco, CEP 36038-330 Juiz de Fora, MG. E-mail: marciocinachi@yahoo.com.br (2)Universidade Estadual Paulista, Faculdade de Ciências Agrárias e Veterinárias, Departamento de Zootecnia, Via de acesso Prof. Paulo Donato

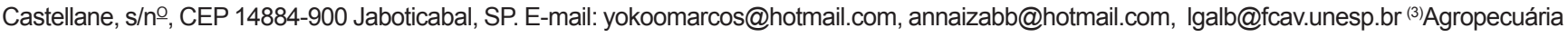
Jacarezinho Ltda., Rodovia Marechal Rondon (SP-300), Km 585, CEP 16880-000 Valparaíso, SP. E-mail: juliana.sesana@yahoo.com.br
\end{abstract}

Resumo - O objetivo deste trabalho foi determinar os fatores que afetam a altura da garupa em diferentes idades, em bovinos Nelore, e estimar a herdabilidade e as correlações genéticas entre esse caractere e as características reprodutivas e de crescimento. Os caracteres avaliados foram: altura da garupa à desmama, altura da garupa ao sobreano, peso à desmama, peso ao sobreano, perímetro escrotal e idade ao primeiro parto. Os fatores considerados foram: ano e mês de nascimento, rebanho, sexo, idade da vaca ao parto e idade do bezerro. Os componentes de variância e covariância foram estimados pela metodologia de máxima verossimilhança restrita, tendo-se utilizado um modelo animal. Todos os efeitos foram significativos para altura de garupa nas diferentes idades. As estimativas de herdabilidade quanto ao efeito genético direto indicaram que as características de crescimento e reprodutivas respondem à seleção, exceto a idade ao primeiro parto. As correlações genéticas entre as características de crescimento foram todas positivas e elevadas, de 0,42 a 0,90 , o que indica que são determinadas em grande parte pelos mesmos conjuntos de genes de ação aditiva. Em razão das baixas magnitudes das estimativas de correlação genética (entre - 0,14 e 0,16 ), a eficiência reprodutiva é pouco influenciada pela seleção quanto à altura de garupa.

Termos para indexação: componentes de variância, correlações genéticas, herdabilidade, idade da vaca, idade do bezerro.

\section{Hip height and its relationships with reproductive and growth traits in Nelore cattle}

\begin{abstract}
The objective of this work was to determine factors that affect hip height at different ages, in Nelore cattle, and to estimate the heritability and the genetic correlation between this character and reproductive and growth traits. The analyzed traits were: hip height at weaning, hip height at post weaning, weight at weaning, weight at post weaning, scrotal circumference, and age at first calving. The analyzed factors were: year and month of birth, herd, sex, age of dam at calving, and calf age. The variance covariance components were estimate by the restrict maximum likelihood methodology using animal model. All effects were significant for hip height at different ages. The heritability estimates for direct genetic effects indicated that the growth and reproductive traits respond to selection, except for age at first calving. The genetic correlations between growth traits were positive and high, from 0.42 to 0.90 , which indicates that they are mostly determined by the same group of genes with additive action. Due to the low magnitude of the estimated genetic correlations, from - 0.14 to 0.16 , the reproductive efficiency is little influenced by selection for hip height.
\end{abstract}

Index terms: variance components, genetic correlations, heritability, age of dam, age of calf.

\section{Introdução}

No Brasil, diversos programas de melhoramento genético têm sido implantados durante a última década, o que tem elevado os índices de produtividade da pecuária de corte. Entretanto, em vários desses programas, são priorizadas as características de crescimento, com ênfase às medidas de peso vivo do animal, sem avaliar a sua associação com outras medidas importantes para um sistema de produção eficiente.

Segundo Rocha et al. (2003), o peso corporal deve ser avaliado em conjunto com outra medida linear de tamanho corporal, como a altura da garupa, para que 
se obtenham resultados confiáveis na determinação do tamanho à maturidade, uma vez que o peso pode apresentar flutuações periódicas. A característica altura da garupa é de fácil mensuração e menos suscetível a variações de meio ambiente. Alguns autores (Riley et al., 2002; Silva et al., 2003; Yokoo et al., 2007) estimaram herdabilidades de magnitude moderada à alta (de 0,30 a 0,67$)$ para o caractere altura da garupa, em zebuínos.

Alguns estudos demonstraram a existência de associação genética entre peso e altura da garupa (Cyrillo et al., 2001; Silva et al., 2003). Assim, a seleção apenas quanto ao peso, em longo prazo pode levar à produção de animais mais altos que, em determinados ambientes, podem ser mais tardios e menos eficientes. Além disso, Yokoo et al. (2010) estimaram, em bovinos da raça Nelore, correlação genética negativa e de magnitude moderada entre altura da garupa e espessura de gordura subcutânea na carcaça.

O conhecimento da associação do peso e da altura com as características reprodutivas é de grande relevância, visto que a fertilidade determina a quantidade de animais produzidos para o mercado, e pode ser considerada como o fator isolado mais importante na determinação da lucratividade da atividade pecuária. Albera et al. (2004) e Kluyts et al. (2007) destacaram a importância econômica de características reprodutivas em sistemas de cria, em comparação às características de produto final, tais como conformação da carne ao abate, rendimento de cortes cárneos, espessura de gordura subcutânea, maciez e marmoreio.

Neste sentido, há a necessidade de se verificar como a altura da garupa em diferentes idades responde à seleção e suas relações com as características de crescimento e de reprodução, atualmente consideradas nos programas de seleção.

O objetivo deste trabalho foi identificar fatores que afetam a altura da garupa em diferentes idades e estimar a herdabilidade e as correlações genéticas entre altura da garupa e características reprodutivas e de crescimento.

\section{Material e Métodos}

Os dados utilizados neste trabalho são provenientes de 110.978 animais da raça Nelore, nascidos entre 1984 e 2003, pertencentes a 12 rebanhos comerciais, localizados nas regiões Sul, Sudeste e Centro-Oeste do Brasil, participantes do programa de melhoramento genético realizado pela Conexão Delta $\mathrm{G}$, associação de pecuaristas que atua em diversas áreas da produção animal e cujos sistemas de produção caracterizam-se pelo ciclo curto. No que se refere ao manejo, os animais são mantidos em regime de pasto e recebem suplementação mineral à vontade; são pesados, avaliados e selecionados periodicamente, com pesagens ao nascimento, à desmama e ao sobreano; as medidas de altura da garupa são realizadas à desmama e ao sobreano, e a medida do perímetro escrotal é efetuada ao sobreano.

Os caracteres avaliados foram: altura da garupa à desmama (Altd) e ao sobreano (Alts), peso à desmama (PD) e ao sobreano (PS), perímetro escrotal (PE) e idade ao primeiro parto (IPP). Para as alturas nas diferentes idades, os registros foram tomados durante quatro anos e, para os demais caracteres, durante 20 anos. A altura foi mensurada com o auxílio de um hipômetro, com o animal contido, tendo-se tomado a medida da ponta do íleo até o solo.

Para se verificar a influência de fatores, tais como ano, mês de nascimento, rebanho, sexo, idade da vaca ao parto e idade do bezerro, sobre as características estudadas, foram realizadas análises de variância pelo método dos quadrados mínimos, com o procedimento GLM do SAS (SAS Institute, 2000). Com base nos resultados dessas análises, foram determinados os efeitos que definiram os grupos de contemporâneos a serem incluídos no modelo misto. Para as características medidas à desmama, o critério de agrupamento para compor os grupos de contemporâneos (GC) foi: animais do mesmo sexo, ano e mês de nascimento, fazenda e grupo de manejo do nascimento à desmama. Para as características tomadas ao sobreano, foram incluídos, na definição dos grupos de contemporâneos, a fazenda e o grupo de manejo ao sobreano, além dos efeitos determinados à desmama. As covariáveis avaliadas foram idade da vaca ao parto e idade do bezerro, quanto às características de crescimento, e idade e peso no momento da medida do perímetro escrotal.

$\mathrm{Na}$ edição dos dados, foram eliminadas as informações de animais cujas medidas estavam a três e meio desvios-padrão acima ou abaixo da média do seu grupo de contemporâneo, para cada característica. Foram excluídos os grupos de contemporâneos que continham apenas animais filhos de um mesmo touro, 
além de grupos de contemporâneos com menos de cinco animais.

Para a estimação dos componentes de (co)variância, empregou-se o programa MTDFREML, descrito por Boldman et al. (1995), com a metodologia de máxima verossimilhança restrita, tendo-se aplicado um algoritmo livre de derivadas. Desta forma, o modelo geral completo usado pode ser representado na forma matricial como:

$$
\mathrm{y}=\mathrm{X} \beta+\mathrm{Z}_{\mathrm{a}} \mathrm{a}+\mathrm{Z}_{\mathrm{m}} \mathrm{m}+\mathrm{Z}_{\mathrm{c}} \mathrm{c}+\varepsilon
$$

em que: y é o vetor das características observadas; $\beta$ é um vetor de efeitos fixos; a é um vetor de efeitos genéticos aditivos diretos; $\mathrm{m}$ é um vetor de efeitos genéticos aditivos maternos; c é um vetor de efeitos de ambiente permanente; $\varepsilon$ é um vetor de efeitos residuais; as matrizes $\mathrm{X}, \mathrm{Z}_{\mathrm{a}}, \mathrm{Z}_{\mathrm{m}}$ e $\mathrm{Z}_{\mathrm{c}}$ representam, respectivamente, as matrizes de incidência dos efeitos $\beta$, a, m e c a y. Admite-se que $\mathrm{E}[\mathrm{y}]=\mathrm{X} \beta, \operatorname{Var}(\mathrm{a})=\mathrm{A} \otimes \Sigma_{\mathrm{a}}, \operatorname{Var}(\mathrm{m})=\mathrm{A} \otimes \Sigma_{\mathrm{m}}, \operatorname{Var}(\mathrm{c})=\mathrm{A} \otimes \Sigma_{\mathrm{c}}$, e $\operatorname{Var}(\varepsilon)=\mathrm{A} \otimes \Sigma_{\varepsilon}$, em que: $\Sigma_{\mathrm{a}}$ é a matriz de covariâncias genéticas aditivas; $\Sigma_{\mathrm{m}}$ é a matriz de covariâncias genéticas maternas; $\Sigma_{\mathrm{c}}$ é a matriz de covariâncias de ambiente permanente; $\Sigma_{\varepsilon}$ é a matriz de covariância residual; A é o numerador da matriz de relações genético-aditivas; I é matriz identidade; e $\otimes$ denota produto direto entre matrizes. Admite-se, ainda, que os vetores c e $\varepsilon$ não são correlacionados com os outros efeitos.

A inclusão dos efeitos aleatórios foi definida por meio do teste de razão de verossimilhança, que se baseia na distribuição de qui-quadrado com g graus de liberdade e probabilidade de erro de $5 \%$, em que g é a diferença em números de parâmetros estimados nos modelos comparados (Dobson, 1990).

\section{Resultados e Discussão}

Na Tabela 1, estão apresentados o número de animais, médias, desvios-padrão, coeficientes de variação, número de touros, vacas e grupos de contemporâneos para as características e covariáveis estudadas.

Todos os efeitos incluídos no modelo influenciaram significativamente $(p<0,05)$ a Altd e Alts, com exceção dos efeitos linear e quadrático da idade da vaca ao parto quanto a Alts (Tabela2). O efeito de interação entre fazenda e mês de nascimento não influenciou significativamente a idade ao primeiro parto, assim como os efeitos linear e quadrático da idade da vaca e idade do bezerro ao sobreano não influenciaram o perímetro escrotal. Os efeitos sistemáticos (sexo, ano e mês de nascimento, fazenda e grupo de manejo do nascimento ao sobreano) são importantes na expressão de uma característica e devem ser considerados em procedimentos de avaliação genética. A correta definição dos grupos contemporâneos é um dos aspectos mais importantes para predizer valores genéticos confiáveis, pois grande parte dos efeitos e suas interações são consideradas nestes grupos.

O resultado evidencia a existência de associação entre a idade da vaca ao parto e a altura da cria à desmama, para ambos os sexos (Figura 1 A). Além disso, os valores da altura à desmama dos machos foram superiores em relação ao das fêmeas. As progênies (machos e fêmeas) de novilhas com três e quatro anos de idade ao parto, bem como as de vacas com idade superior a 13 anos, apresentaram alturas ao desmame inferiores às de filhos de vacas com idade ao parto entre 5 e 12 anos. De maneira geral, os filhos de vacas com idade entre sete e nove anos de idade apresentaram valores de altura à desmama situados no platô da curva, porém, o pico foi

Tabela 1. Número de animais (N), médias, desvios-padrão (DP), coeficientes de variação (CV), número de touros (NT) e vacas $(\mathrm{NV})$ e número de grupos de contemporâneos (NGC) para as características e covariáveis estudadas.

\begin{tabular}{|c|c|c|c|c|c|c|c|}
\hline Variável $^{(1)}$ & $\mathrm{N}$ & Média & DP & $\mathrm{CV}(\%)$ & NT & NV & NGC \\
\hline Altura à desmama $(\mathrm{cm})$ & 18.011 & 115,98 & 4,34 & 3,74 & 218 & 15.947 & 321 \\
\hline Altura ao sobreano $(\mathrm{cm})$ & 7.659 & 132,86 & 5,80 & 4,36 & 179 & 7.375 & 222 \\
\hline Peso à desmama (kg) & 110.978 & 168,53 & 24,97 & 14,82 & 511 & 48.319 & 2.515 \\
\hline Peso ao sobreano $(\mathrm{kg})$ & 34.252 & 265,28 & 39,78 & 15,00 & 386 & 21.099 & 1.383 \\
\hline Perímetro escrotal $(\mathrm{cm})$ & 13.270 & 25,60 & 3,16 & 12,34 & 333 & 10.141 & 573 \\
\hline IPP (dias) & 21.248 & $1.063,55$ & 82,44 & 7,75 & 276 & 14.488 & 1.790 \\
\hline IDV (anos) & 128.916 & 5,79 & 3,06 & 52,76 & - & - & - \\
\hline IDBd (dias) & 115.790 & 193,84 & 21,25 & 10,96 & - & - & - \\
\hline IDBs (dias) & 36.347 & 503,21 & 36,22 & 7,20 & - & - & - \\
\hline PCOV $(\mathrm{kg})$ & 53.930 & 267,36 & 47,57 & 17,79 & - & - & - \\
\hline
\end{tabular}


mais evidente para os machos, e a curva mais plana para as fêmeas. Estudos sobre a influência da idade da vaca sobre a altura à desmama são escassos, entretanto, essa diferença é semelhante à observada em escores visuais e ganho médio diário no período pré-desmama, em animais da raça Nelore (Jorge Júnior et al., 2004; Bocchi $\&$ Albuquerque, 2005). Apesar de o efeito de sexo estar incluído no grupo de contemporâneos, os resultados deste trabalho indicam que o efeito da idade da vaca ao parto sobre a altura à desmama mostrou diferentes tendências para machos e fêmeas, o que torna necessária a correção para cada sexo separadamente.

A altura do bezerro à desmama aumentou linearmente com o avanço da idade (Figura 1 B), e os bezerros desmamados com mais idade foram mais altos, conforme o esperado. Esse fato também acontece com o peso, pois, geralmente, os animais mais velhos são mais pesados. Quanto à altura ao sobreano, constatou-se efeito quadrático significativo da idade do animal (Figura $1 \mathrm{C}$ ). Com esses resultados, fica claro que medidas corporais são dependentes também dos efeitos da idade do animal e devem ser corrigidas para essa fonte de variação, na predição dos valores genéticos.

Os modelos mais adequados, quanto aos efeitos aleatórios considerados para cada característica avaliada, e suas respectivas estimativas de herdabilidade e componentes de variância podem ser observados na Tabela 3. O valor estimado de herdabilidade da altura, medida à desmama e ao sobreano, indica a existência de variabilidade genética. Estimativas superiores da altura à desmama $(0,65)$ e ao sobreano $(0,87)$ foram encontradas

Tabela 2. Resumo da análise de variância quanto à altura da garupa à desmama (Altd) e ao sobreano (Alts) de animais da raça Nelore.

\begin{tabular}{|c|c|c|c|c|}
\hline \multirow[t]{2}{*}{ Fonte de variação $^{(1)}$} & \multicolumn{2}{|r|}{ Altd } & \multicolumn{2}{|r|}{ Alts } \\
\hline & $\mathrm{GL}^{(2)}$ & Quadrado médio & GL & Quadrado médio \\
\hline Rebanho & 3 & $109,41 * *$ & 4 & $8556,10 * *$ \\
\hline Ano de nascimento & 3 & $289,24 * *$ & 2 & 7741,02 \\
\hline Mês de nascimento & 10 & $1433,96 * *$ & 7 & 197,03 \\
\hline Sexo & 1 & $105,99 * *$ & 1 & $54.518,52$ \\
\hline IDV & - & - & 1 & $45,53 \mathrm{~ns}$ \\
\hline Interação IDV e sexo & 2 & $3508,31 * *$ & - & - \\
\hline $\mathrm{IDV}^{2}$ & - & - & 1 & $2,26 \mathrm{~ns}$ \\
\hline Interação IDV ${ }^{2}$ e sexo & 2 & $2618,02 * *$ & - & - \\
\hline IDB & 1 & $78,46^{*}$ & 1 & $304,49 * *$ \\
\hline $\mathrm{IDB}^{2}$ & 1 & $0,49 \mathrm{~ns}$ & 1 & $170,57 * *$ \\
\hline Residual & 17.987 & 14,32 & 7.639 & 14,40 \\
\hline
\end{tabular}

porVargas etal.(1998, 2000), em animais da raça Brahman, com uso, entretanto, de conjuntos de dados com menor número de animais. As estimativas de herdabilidades,
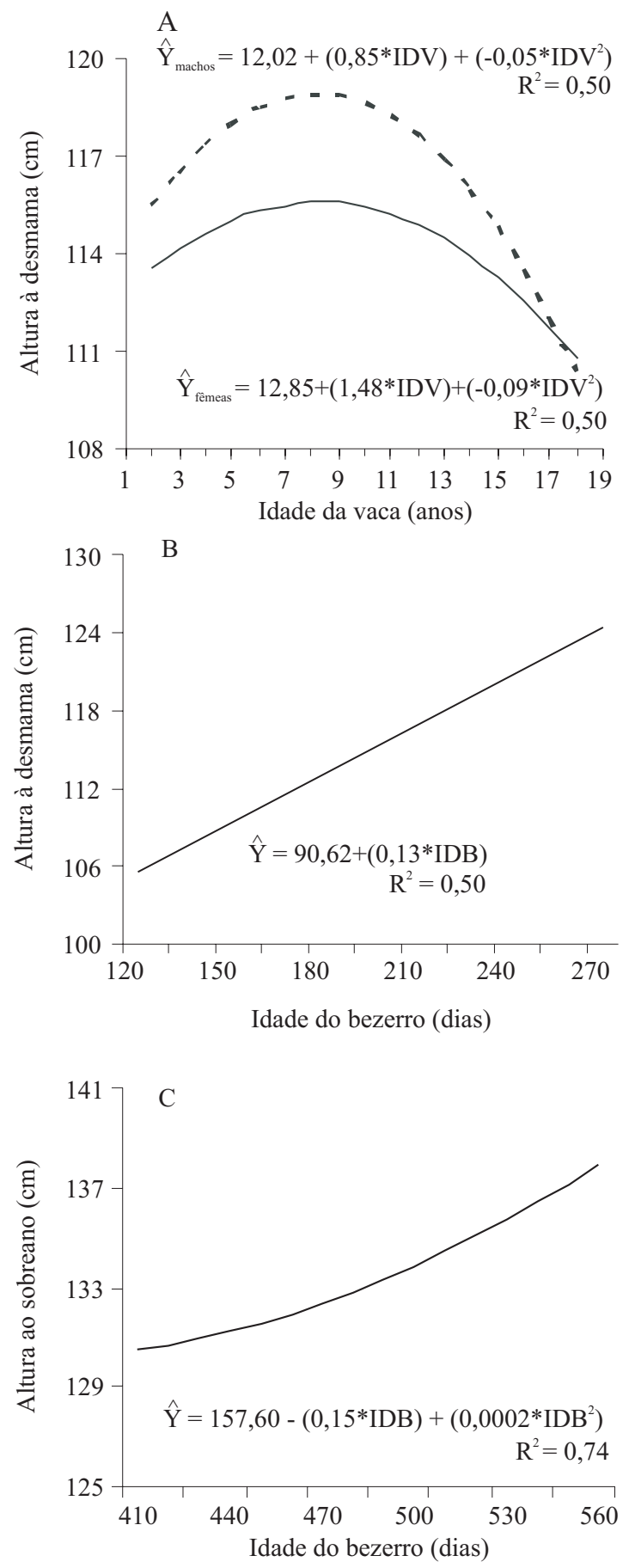

Figura 1. Estimativas da altura da garupa à desmama em machos e fêmeas, em função da idade da vaca (IDV), e da altura à desmama e ao sobreano, em função da idade do bezerro (IDB), em animais da raça Nelore. 
obtidas quanto à altura nas diferentes idades, foram de alta magnitude e semelhantes. Considerando-se os erros-padrão obtidos nas análises unicaracterística (Tabela $3)$, os valores das estimativas se sobrepõem. A estimativa de herdabilidade quanto à altura estimada à desmama é inferior às encontradas por Vargas et al. (2000), em um rebanho da raça Brahman; porém, é superior à encontrada por Bourdon \& Brinks (1986), em um rebanho da raça Hereford. No Brasil, são encontrados poucos estudos relativos à altura da garupa em raças zebuínas; entre eles, os de Cyrillo et al. (2001) e Silva et al. (2003) relataram valores de 0,31 e 0,30 , respectivamente, inferiores aos do presente trabalho quanto à altura ao sobreano. Entretanto, Yokoo et al. (2007) reportaram estimativa de herdabilidade semelhante $(0,63)$ deste trabalho, para a raça Nelore ao sobreano.

Em sistemas de produção em que animais de estatura mediana são mais eficazes, como os de propriedades com escassez de alimento e água, cujos produtores desejam animais de ciclo de produção mais curto, a seleção de animais como pais de futuras gerações com valores genéticos próximos de zero, quanto à altura nas diferentes idades, poderá ser utilizada como ferramenta em programas de melhoramento animal, visto que a altura é uma característica que deverá responder rapidamente à seleção massal. As estimativas de herdabilidade direta quanto aos pesos elevaram-se com o avanço da idade.
Esse comportamento de incremento para as estimativas de herdabilidade direta após a desmama também foram observados por Albuquerque \& Meyer (2001), Dias et al. (2005) e Yokoo et al. (2007).

De acordo com o teste de razão de verossimilhança (Dobson, 1990), o modelo mais adequado quanto ao efeito aleatório para o peso ao soberano foi o que incluía apenas os efeitos aleatórios direto e materno, além do residual. Neste trabalho, as estimativas de herdabilidade materna quanto ao peso foram semelhantes entre a desmama e o sobreano, considerando os respectivos erros-padrão. Entretanto, na literatura, alguns trabalhos têm relatado diminuição na magnitude das estimativas de herdabilidade materna entre essas idades (Albuquerque \& Meyer, 2001; Dias et al., 2005).

A habilidade materna é de fundamental importância no desempenho dos bezerros, desde a fase pré-natal, em razão das diferenças placentárias, até a fase pós-natal, em consequência dos cuidados proporcionados à cria e da produção de leite da mãe. Essa influência foi evidenciada no peso, em que a contribuição do efeito materno para a variação fenotípica total foi de 7 e $8 \%$ para o peso à desmama e ao sobreano, respectivamente (Tabela 3). Alencar (1987) verificou que a produção de leite de vacas é responsável por 26,2 e $20,3 \%$ do ganho em peso, do nascimento ao desmame de seus bezerros, nas raças Canchim e Nelore, respectivamente.

Tabela 3. Estimativas dos componentes de variâncias e dos parâmetros genéticos das características estudadas, em análises unicaracteristica.

\begin{tabular}{|c|c|c|c|c|c|c|c|}
\hline \multirow[t]{2}{*}{ Característica } & \multicolumn{4}{|c|}{ Componentes de variância $^{(1)}$} & \multicolumn{3}{|c|}{ Parâmetros genéticos ${ }^{(2)}$} \\
\hline & $\begin{array}{ll}2 \\
a\end{array}$ & $\begin{array}{l}2 \\
\mathrm{~m} \\
\end{array}$ & $\begin{array}{ll}2 \\
\mathrm{c} \\
\end{array}$ & $\begin{array}{ll}2 \\
\mathrm{e}\end{array}$ & $\mathrm{h}_{\mathrm{d}}^{2}$ & $\mathrm{~h}_{\mathrm{m}}^{2}$ & $\mathrm{c}^{2}$ \\
\hline Altura à desmama (kg) & 6,57 & - & - & 3,81 & $0,63 \pm 0,04$ & - & - \\
\hline Altura ao sobreano $(\mathrm{cm})$ & 5,82 & - & - & 3,51 & $0,62 \pm 0,07$ & - & - \\
\hline Peso à desmama (kg) & 52,54 & 17,90 & 38,38 & 141,82 & $0,21 \pm 0,01$ & $0,07 \pm 0,01$ & $0,15 \pm 0,01$ \\
\hline Peso ao sobreano $(\mathrm{kg})$ & 119,07 & 67,40 & - & 276,69 & $0,24 \pm 0,02$ & $0,08 \pm 0,01$ & - \\
\hline Perímetro escrotal (cm) & 2,98 & - & - & 3,10 & $0,49 \pm 0,04$ & - & - \\
\hline Idade ao primeiro parto (dias) & 147,25 & - & - & $1.703,63$ & $0,08 \pm 0,02$ & - & - \\
\hline
\end{tabular}

${ }^{(1)} \sigma_{\mathrm{a}}^{2}$, variância genética aditiva direta; $\sigma_{\mathrm{m}}^{2}$, variância genética aditiva materna; $\sigma_{\mathrm{c}}^{2}$, variância de ambiente permanente materno; $\sigma_{\mathrm{e}}^{2}$, variância residual. ${ }^{(2)} h_{\mathrm{d}}^{2}$, herdabilidade direta; $\mathrm{h}_{\mathrm{m}}^{2}$, herdabilidade materna; e $\mathrm{c}^{2}$, variância de ambiente permanente materno, como proporção da variância fenotípica.

Tabela 4. Estimativas de herdabilidade (diagonal de Altd a PE $\mathrm{a}_{\mathrm{a}}$ ), correlações genéticas (acima da diagonal) e fenotípicas (abaixo da diagonal), entre as características estudadas.

\begin{tabular}{|c|c|c|c|c|c|c|c|c|}
\hline Característica $^{(1)}$ & Altd & Alts & PD & PS & IPP & $\mathrm{PE}_{\mathrm{a}}$ & $\mathrm{PE}_{\mathrm{h}}$ & $\mathrm{PE}_{c}$ \\
\hline$\overline{\text { Altd }}$ & $0,53^{*}$ & 0,90 & 0,87 & 0,58 & $-0,03$ & $-0,01$ & $-0,01$ & 0,12 \\
\hline Alts & 0,52 & $0,68^{*}$ & 0,42 & 0,50 & 0,16 & $-0,14$ & $-0,13$ & $-0,04$ \\
\hline PD & 0,64 & 0,44 & $0,24 *$ & 0,88 & $-0,09$ & $-0,15$ & $-0,13$ & 0,11 \\
\hline PS & 0,49 & 0,47 & 0,65 & $0,25^{*}$ & $-0,16$ & $-0,13$ & $-0,11$ & 0,20 \\
\hline IPP & 0,57 & 0,17 & $-0,03$ & $-0,06$ & $0,05^{*}$ & 0,08 & $-0,23$ & $-0,22$ \\
\hline $\mathrm{PE}$ & $-0,06$ & $-0,05$ & 0,02 & 0,01 & 0,40 & $0,50 *$ & $0,46^{*}$ & $0,44^{*}$ \\
\hline
\end{tabular}

${ }^{(1)}$ Altd, altura da garupa à desmama; Alts, altura da garupa ao sobreano; PD, peso à desmama; PS, peso ao sobreano; IPP, idade ao primeiro parto; $\mathrm{PE}$, perímetro escrotal; $\mathrm{PE}_{\mathrm{a}}$, perímetro escrotal ajustado para peso; $\mathrm{PE}_{\mathrm{b}}$, perímetro escrotal ajustado para peso e idade; $\mathrm{PE}_{\mathrm{c}}$, perímetro escrotal ajustado para idade. *Médias das estimativas das herdabilidades de todas as análises bicaracterísticas. 
Altas estimativas de herdabilidade quanto ao perímetro escrotal, como encontrado neste trabalho (Tabela 3 e 4), têm sido descritas na literatura para a raça Nelore (Cyrillo et al., 2001; Pereira et al., 2002; Yokoo et al., 2007). Para idade ao primeiro parto, a estimativa de herdabilidade foi de magnitude baixa (Tabela 3 e 4) e está de acordo com resultados encontrados na literatura para animais da raça Nelore (Dias et al., 2004; Forni \& Albuquerque, 2005; Silva et al., 2005). Contudo, alguns autores relataram valores de herdabilidade de moderados a altos (Westhuizen et al., 2001; Bertazzo et al., 2004).

Para todas as características analisadas, os valores de herdabilidade estimados em análise bicaracterística (Tabela 4) foram próximos aos estimados por análise unicaracterística (Tabela 3), com exceção dos caracteres altura e peso à desmama. Nesses casos, as estimativas de herdabilidade, obtidas nas análises bicaracterísticas, estavam fora da amplitude, quando se considerou o erro-padrão nas análises unicaracterísticas.

As correlações genéticas entre os caracteres de crescimento foram todas positivas de magnitudes moderadas a altas, o que indica que são determinadas, em grande parte, pela ação aditiva dos mesmos conjuntos de genes (Tabela 4). Portanto, a seleção quanto ao peso, a depender da intensidade de seleção aplicada, pode em médio e longo prazo levar a um aumento na estatura dos animais. Porém, como a correlação não é igual à unidade, devem-se associar essas características, em programas de melhoramento genético animal, por meio de um índice de seleção, para a obtenção de animais pesados e de altura moderada. Uma alternativa para os programas de melhoramento é o monitoramento da estatura dos animais no rebanho, assim como é realizado com o peso ao nascimento.

As correlações genéticas entre as características de crescimento e perímetro escrotal foram de baixa magnitude, o que indica que a seleção quanto a peso e altura exerce pouca influência no perímetro escrotal. Alguns autores relataram valores de estimativas de correlação genética próximas em magnitude, mas em direções opostas às encontradas neste trabalho, quanto à altura da garupa ao sobreano e perímetro escrotal, para raças zebuínas (Vargas et al., 1998; Yokoo et al., 2007). Também, em relação à raça Nelore, Cyrillo et al. (2001) relataram correlação genética positiva e moderada entre altura e perímetro escrotal.
A correlação genética baixa e negativa entre os pesos e o perímetro escrotal, neste trabalho, pode ser explicada pelo fato de as medidas do perímetro escrotal estarem ajustadas para a covariável peso e idade do animal. Observou-se que as correlações genéticas entre os pesos e o perímetro escrotal corrigidas para peso e idade, ao invés de somente para a idade, passaram de positivas a negativas, porém permaneceram com baixa magnitude. Essa mesma mudança da correlação genética entre o perímetro escrotal e a altura à desmama foi observada quando o perímetro escrotal foi ajustado quanto ao peso, o que não ocorreu para a altura ao sobreano. São necessários estudos para verificar a associação genética entre a altura, pesos corporais e perímetro escrotal para se evitar respostas correlacionadas indesejáveis, como aumento da altura da garupa, com uso do perímetro escrotal como critério de seleção, sem ajustá-lo ao peso corporal.

A correlação genética entre idade ao primeiro parto e perímetro escrotal foi baixa e negativa, o que está de acordo com o desejável. Boligon et al. (2007) encontraram estimativa similar $(-0,23)$. Da mesma forma, Forni \& Albuquerque (2005) analisaram as características de perímetro escrotal e dias para o primeiro parto e encontraram estimativa semelhante, $o$ que demonstra que a seleção de reprodutores, quanto ao maior perímetro escrotal, fornece, em resposta correlacionada, menor idade ao primeiro parto de sua progênie.

As estimativas de correlações genéticas entre alturas (alturas à desmama e ao sobreano) e idade ao primeiro parto foram baixas, tendo-se elevado entre o período da desmama e o de sobreano. Silva et al. (2003) avaliaram animais da raça Nelore e sugeriram que a seleção quanto ao crescimento, medida na altura da garupa, pouco poderá afetar a precocidade das novilhas. Entretanto, Vargas et al. (1998) relataram que a seleção de touros da raça Brahman, quanto à altura da garupa, não afetou o perímetro escrotal, porém trouxe prejuízo para a característica idade à puberdade. Para os pesos à desmama e ao sobreano, observaram-se correlações genéticas favoráveis com a idade ao primeiro parto, embora de baixas magnitudes, o que indica que novilhas de maior velocidade de ganho de peso tendem a alcançar idade ao primeiro parto mais precocemente.

Todas as correlações fenotípicas entre a altura da garupa, em diferentes idades, e as características de 
crescimento foram inferiores às correlações genéticas e de magnitudes medianas. As correlações fenotípicas entre essas alturas e as características reprodutivas foram superiores às correlações genéticas, com exceção da altura ao sobreano e idade ao primeiro parto.

\section{Conclusões}

1. Todos os fatores avaliados - ano e mês de nascimento, rebanho, sexo, idade da vaca ao parto e idade do bezerro - afetam a altura da garupa.

2. As estimativas de herdabilidade indicam que as características de crescimento e reprodutivas, exceto idade ao primeiro parto, respondem à seleção.

3. As correlações genéticas entre as características de crescimento e a altura da garupa são elevadas, mas a eficiência reprodutiva é pouco afetada pela altura da garupa.

\section{Agradecimentos}

Ao Conselho Nacional de Desenvolvimento Científico e Tecnológico e à Fundação de Amparo à Pesquisa do Estado de São Paulo, pelo apoio financeiro. À Conexão Delta $\mathrm{G}$, pela parceria na realização deste trabalho.

\section{Referências}

ALBERA, A.; CARNIER, P.; GROEN, A.F. Definition of a breeding goal for the Piemontese breed: economic and biological values and their sensitivity to production circumstances. Livestock Production Science, v.89, p.67-78, 2004.

ALBUQUERQUE, L.G.; MEYER, K. Estimates of direct and maternal genetic effects for weights from birth to 600 days of age in Nelore cattle. Journal of Animal Breeding and Genetics, v.118, p.83-92, 2001.

ALENCAR, M.M. Efeitos da produção de leite sobre o desenvolvimento de bezerros Canchim. Revista Brasileira de Zootecnia, v.16, p.1-13, 1987.

BERTAZZO, R.P.; FREITAS, R.T.F.; GONÇALVES, T.M.; PEREIRA, I.G.; ELER, J.P.; FERRAZ, J.B.S.; OLIVEIRA, A.I.G.; ANDRADE, I.F. Parâmetros genéticos de longevidade e produtividade de fêmeas da raça Nelore. Revista Brasileira de Zootecnia, v.33, p.1118-1127, 2004.

BOCCHI, A.L.; ALBUQUERQUE, L.G. Efeito da idade da vaca e da data juliana de nascimento sobre o ganho médio diário de bezerros de corte no período pré-desmame. Arquivo Brasileiro de Medicina Veterinária e Zootecnia, v.57, p.524-532, 2005.

BOLDMAN, K.G.; KRIESE, L.A.; VAN VLECK, L.D.; VAN TASSELL, C.P.; KACHMAN S.D. A manual for use of
MTDFREML: a set of programs to obtain estimates of variance and covariances [DRAFT]. Lincoln: Agricultural Research Service, 1995. 120p.

BOLIGON, A.A.; RORATO, P.R.N.; ALBUQUERQUE, L.G. Correlações genéticas entre medidas de perímetro escrotal e características produtivas e reprodutivas de fêmeas da raça Nelore. Revista Brasileira de Zootecnia, v.36, p.565-571, 2007.

BOURDON, R.M.; BRINKS, J.S. Scrotal circumference in yearling Hereford bulls: adjustment factors, heritabilities and genetic, environmental and phenotypic relationships with growth traits. Journal of Animal Science, v.62, p.958-967, 1986.

CYRILLO, J.N. dos S.G.; RAZOOK, A.G.; FIGUEIREDO, L.A. de; BONILHANETO, L.M.; MERCADANTE, M.E.Z.; TONHATI, H. Estimativas de tendências e parâmetros genéticos do peso padronizado aos 378 dias de idade, medidas corporais e perímetro escrotal de machos Nelore de Sertãozinho, SP. Revista Brasileira de Zootecnia, v.30, p.56-65, 2001.

DIAS, L.T.; ALBUQUERQUE, L.G.; TONHATI, H.; TEIXEIRA, R. de A. Estimação de parâmetros genéticos para peso em diferentes idades para animais da raça Tabapuã. Revista Brasileira de Zootecnia, v.34, p.1914-1919, 2005.

DIAS, L.T.; EL FARO, L.; ALBUQUERQUE, L.G. Efeito da idade de exposição de novilhas à reprodução sobre estimativas de herdabilidade da idade ao primeiro parto em bovinos Nelore. Arquivo Brasileiro de Medicina Veterinária e Zootecnia, v.56, p.370-373, 2004.

DOBSON, A.J. An introduction to generalized linear models. Melbourne: Chapman and Hall, 1990. 174p.

FORNI, S.; ALBUQUERQUE, L.G. Estimates of genetic correlations between days to calving and reproductive and weight traits in Nelore cattle. Journal of Animal Science, v.83, p.1511-1515, 2005.

JORGE JÚNIOR, J.; DIAS, L.T.; ALBUQUERQUE, L.G. Fatores de correção de escores visuais de conformação, precocidade e musculatura, à desmama, para idade da vaca ao parto, data juliana de nascimento e idade à desmama em bovinos da raça Nelore. Revista Brasileira de Zootecnia, v.33, p.2044-2053, 2004.

KLUYTS, J.F.; NESER, F.W.C.; BRADFIELD, M.J. Derivation of economic values for the Simmentaler breed in South Africa. South African Journal of Animal Science, v.37, p.107-121, 2007.

PEREIRA, E.; ELER, J.P.; FERRAZ, J.B.S. Análise genética de características reprodutivas na raça Nelore. Pesquisa Agropecuária Brasileira, v.37, p.703-708, 2002.

RILEY, D.G.; CHASE JUNIOR, C.C.; HAMMOND, A.C.; WEST, R.L.; JOHNSON, D.D.; OLSON, T.A.; COLEMAN, S.W. Estimated genetic parameters for carcass traits of Brahman cattle. Journal of Animal Science, v.80, p.955-962, 2002.

ROCHA, E.D.; ANDRADE, V.J.; EUCLIDES FILHO, K.; NOGUEIRA E.; FIGUEIREDO, G.R. Tamanho de vacas Nelore adultas e seus efeitos no sistema de produção de gado de corte. Arquivo Brasileiro de Medicina Veterinária e Zootecnia, v.55, p.273-283, 2003.

SAS INSTITUTE. SAS/STAT software: changes and enhancements through release. Version 8.2. Cary: SAS Institute, 2000. 
SILVA, J.A. de V.; DIAS, L.T.; ALBUQUERQUE, L.G. Estudo genético da precocidade sexual de novilhas em um rebanho Nelore. Revista Brasileira de Zootecnia, v.34, p.1568-1572, 2005.

SILVA, J.A. de V.; VAN MELIS, M.H.; ELER, J.P.; FERRAZ, J.B.S. Estimação de parâmetros genéticos para probabilidade de prenhez aos 14 meses e altura na garupa em bovinos da raça Nelore. Revista Brasileira de Zootecnia, v.32, p.1141-1146, 2003.

VARGAS, C.A.; ELZO, M.A.; CHASE, C.C.; CHENOWETH, P.J.; OLSON, T.A. Estimation of genetic parameters for scrotal circumference, age at puberty in heifers, and hip height in Brahman cattle. Journal of Animal Science, v.76, p.2536-2541, 1998.

VARGAS, C.A.; ELZO, M.A.; CHASE, C.C.; OLSON, T.A Genetic parameters and relationships between hip height and weight in Brahman cattle. Journal of Animal Science, v.78, p.3045-3052, 2000.
WESTHUIZEN, R.R. van der; SCHOEMAN, S.J.; JORDAAN, G.F.; WYK, J.B. van. Heritability estimates derived from threshold analyses for reproduction and stayability traits in a beef cattle herd. South African Journal of Animal Science, v.31, p.25-32, 2001.

YOKOO, M.J.; ALBUQUERQUE, L.G.; LÔBO, R.B.; SAINZ, R.D.; CARNEIRO JÚNIOR, J.M.; BEZERRA, L.A.F.; ARAUJO, F.R. da C. Estimativas de parâmetros genéticos para altura do posterior, peso e circunferência escrotal em bovinos da raça Nelore. Revista Brasileira de Zootecnia, v.36, p.1761-1768, 2007.

YOKOO, M.J.; LOBO, R.B.; ARAUJO, F.R.C.; BEZERRA, L.A.F.; SAINZ, R.D.; ALBUQUERQUE, L.G. Genetic associations between carcass traits measured by real-time ultrasound and scrotal circumference and growth traits in Nelore cattle. Journal of Animal Science, v.88, p.52-58, 2010.

Recebido em 3 de novembro de 2009 e aprovado em 7 de maio de 2010 